\title{
Structure of wave-particle resonances and Alfvén mode saturation
}

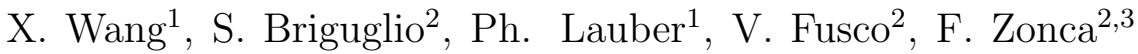

1. Max Planck Institut fur Plasmaphysik, Garching, Germany

2. Associazione Euratom-ENEA sulla Fusione, C.P. 65 - I-00044 - Frascati, Italy

3. Institute for Fusion Theory and Simulation, Zhejiang

University, Hangzhou, People's Republic of China

\begin{abstract}
The dynamics of beta-induced Alfvén eigenmodes driven by anisotropic co-passing or counterpassing fast ions, in a low-shear magnetic equilibrium, is investigated by self-consistent hybrid MHD-particle simulations with the XHMGC code. Though the modes exhibit similar structure and frequency in both cases, and the linear growth rate is $10 \%$ larger for counter-passing ions than for co-passing ions, the nonlinear saturation amplitude is much larger in co-passing case. Moreover, different scalings for the saturation amplitude with increasing growth rates are observed in the two cases. It is shown that these differences are caused by the different radial dependence of resonant frequencies of co-passing and counter-passing fast ions: flat in the former case, steep in the latter one.
\end{abstract}




\section{INTRODUCTION}

Shear-Alfvén modes can be driven unstable, in burning plasmas, by the resonant interaction with energetic ions produced by additional heating or nuclear fusion reactions. These particles are indeed characterized by velocities of the order of the Alfvén speed, the typical propagation velocity of those modes. The Alfvénic fluctuations generated in this way can in turn affect the confinement of energetic ions, preventing their complete thermalisation in the central region of the plasma and, possibily, increasing the thermal load on the material wall surrounding it. The assessment of energetic particle confinement properties in next generation fusion experiments then strongly relies on the comprehension of Alfvén mode dynamics, with regard both to the linear stability properties (which modes are expected to be driven unstable) and the nonlinear saturation mechanisms (which saturation level is expected for the mode amplitude and which effect on the fast ion confinement).

The relevance of these issues has motivated a very rich literature on the side of linear stability investigation [1-6]. Studies of the nonlinear saturation of Alfvén modes include theoretical and numerical analyses, as well as comparisons of the corresponding predictions with the experimental observations [4, 5, 7, 8]. In general, the saturation mechanisms of Alfvén eigenmodes (AE) can be classified into two major categories: fluid mode-mode coupling and nonlinear wave-particle interactions. Although the former can play a crucial role in multi-scale dynamics of burning plasmas [2, 9], we will focus, in the present paper, on the latter category, motivated by the relevance of the resonant drive on the Alfvénic fluctuation spectrum in fusion devices. Most of nonlinear studies concerning wave-particle dynamics have been focused on two extreme limits: weak Toroidal Alfvén Eigenmodes [10] (TAEs) close to marginal stability and Energetic Particle Modes [11] (EPMs) strongly driven by large gradients of fast ion pressure. It has been shown [12 14 that the former modes saturate because of phase mixing of resonant particles trapped in the potential well of the wave (i.e., gradient flattening on the length scale of the mode) [12], reducing the wave-particle power transfer. In this near-marginal stability regime, the radial excursion of fast ions due to the mode-particle interaction is so limited that it does not allow resonant particles to perceive any of the (equilibrium or fluctuation induced) non-uniformities characterising the system. This fact may result in the formation of phase-space holes and clumps and adiabatic frequency chirping due to resonant frequencies following hole and clump propagation, as 
discussed in Ref.[[14-17]]. Strongly driven EPMs saturate instead because of large radial displacement of resonant particles (a potentially large fraction of the whole population), with consequent macroscopic distortion of the energetic particle pressure profile. It has been shown [18] that this mechanism, with an outwardly moving front of pressure gradient, can give rise to avalanches: oscillations of the shear-Alfvén continuous spectrum are driven progressively unstable by the drifting free energy source. In this non-perturbative, stronglydriven regime, the radial excursion of resonant particles is so essential that it self-consistently determines the nonlinear mode structure evolution.

The transition between these two limiting behaviours has been less explored, though it is likely that burning plasmas will be characterised by shear-Alfvén modes in an intermediate dynamic regime. In Ref. [19, this issue has been investigated by applying test-particle diagnostics to the analysis of nonlinear resonant particle dynamics. Two different cases have been considered in that paper: a $n=6$ TAE (with $n$ being the toroidal mode number) in a low magnetic shear equilibrium and a $n=2$ EPM in a plasma equilibrium with larger shear. It has been shown that in the former case saturation is due to a mechanism called radial decoupling [19, 20], with the radial mode structure playing a major role; in the latter, saturation is caused by a mechanism called resonance detuning [19, 20], dictated by the finite radial width of the region where particles can effectively resonate with the mode.

In the present paper, we want to show that these two different mechanisms can occur even for a single plasma equilibrium and a particular Alfvén eigenmode with a particular toroidal mode number $n$, in situations that, from a macroscopic point of view (that is, looking at the mode-particle interaction integrated over the whole energetic-particle distribution) may appear to be characterised by analogous linear behaviour. To this aim, we will present the results of a numerical investigation related to the linear and nonlinear evolution of beta induced Alfvén eigenmodes (BAEs). The experimental observation [21, 22] of these lowfrequency modes (frequencies lower than that of TAEs, and of the order of thermal-ion diamagnetic or transit frequency), has demonstrated that they can be deleterious for fastion confinement. Thus, BAE's dynamics is an important topic for the understanding of magnetically confined plasma behaviour, per se.

The investigation of BAEs requires a proper kinetic treatment of wave-particle interactions, both for energetic and thermal particles [23, 24]. In the frequency range where kinetic thermal-ion response affects the structure of the shear-Alfvén continuum and influ- 
ence plasma dynamics [1], there is a continuous transition between various shear-Alfvén wave (SAW) branches, as predicted theoretically [11, 24 30] and confirmed experimentally [21, 3139]. A theoretical analysis of the linear stability of BAEs has been carried out [40], in the framework of the so-called generalized fishbone like dispersion relation (GFLDR) [11, 27, 4143], while, in that paper, only some aspects of the nonlinear dynamics of these modes has been explored.

In the present work, we will investigate the nonlinear wave-particle interactions for BAEs driven unstable by fast ions characterised by anisotropic Maxwellian distribution function, by means of numerical simulations performed by a hybrid magnetohydrodynamics-particle code (XHMGC) [44 46]. We will analyze two cases with co-passing and counter-passing energetic ions, respectively. The results can be summarized as follows. While the co-passing and counter-passing ion cases present similar linear-dynamics mode features (in particular, similar mode frequencies and mode radial structures), the mode saturation amplitude, in the weak-drive limit, is significantly larger for the co-passing fast ion case than the counterpassing one, in spite of the $10 \%$ larger growth rate observed in the latter case. Moreover, for co-passing ions, a linear dependence of the saturation amplitude on the mode growth rate is found; for counter-passing ions, a quadratic scaling is obtained in the weak-drive limit, along with a transition to a linear scaling in the strong-drive limit. In the latter limit saturation amplitudes for co-passing and counter-passing ion cases converge to the same value. In order to explain these differences a detailed analysis of the resonant interactions between mode and fast ions is performed by looking at phase-space slices characterised by fixed values of constants of motions. Mode-particle interactions have then a "local" character, in the sense that particles belong to the same phase space slice throughout the nonlinear dynamics; and each phase space slice yields its own contribution to mode dynamics; the time evolution of such contribution can be analysed independently of the others. Considering the phase space slice where the maximum resonant interaction is localised, it is shown that resonance frequencies of co-passing and counter-passing fast ions have different radial dependence, because of their different orbits in the non-uniform tokamak equilibrium. Although mode saturation is reached, in both cases, because of resonant-ion density flattening, in the copassing ion case, the resonance width is very large, and the width of the flattening region required for reaching mode saturation is of the order of the smaller mode width (saturation due to radial decoupling). For counter-passing ions, in the low growth rate limit, the res- 
onance width is much smaller than the mode radial extension and represents the relevant limit for the flattening region at saturation (saturation due to resonance detuning); for larger growth rates, a transition to the radial decoupling regime is observed. On the basis of a simple model, we find that resonance detuning and radial decoupling regimes correspond to different expressions of the saturation amplitude on mode width, growth rate and radial slope of the resonance frequency. These expressions reproduce quite well the ratio between saturation amplitudes in the co-passing and counter-passing ion simulations, as well as the scaling of such amplitudes with the growth rate.

The paper is organised as follows. In section II], we describe the numerical method and the main elements of the system considered in our BAE simulations, including thermal plasma and co-passing and counter-passing fast ion populations. In section III, linear dynamics of resonantly excited BAE is analysed, with respect both to macroscopic mode characteristics and microscopic resonant-particle features. In section IV, nonlinear dynamics is discussed along with the different properties of the saturation state in the two considered cases. The heuristic model is presented in section V. Finally, summary and conclusions are presented in section VI.

\section{NUMERICAL METHOD AND SIMULATION PARAMETERS}

The extended version of the nonlinear hybrid magnetohydrodynamics-gyrokinetic code (XHMGC) 44 46] has been used to simulate self-consistenly the BAE driven by fast ions characterized by anisotropic-Maxwellian distribution functions. Within this hybrid model, the fluid response of the thermal background plasma is described by a set of $O\left(\epsilon^{3}\right)$-reduced MHD equations for a low- $\beta$ plasma, and the fast-ion and thermal-ion kinetic dynamics enter via the pressure tensors, which are computed by solving the gyrokinetic equations, in the drift-kinetic limit, by particle-in-cell techniques. Finite-Larmor-radius effects are ignored, while finite-orbit widths are taken into account in order to retain resonant wave-particle dynamics associated with guiding-center magnetic-curvature drift in toroidal geometry. Assuming cold electrons, ideal-MHD Ohm's law $\left(E_{\|}=0\right)$ could be considered; but XHMGC requires a small resistivity, for numerical reasons. In the present study, we ignore thermal-ion diamagnetic effects by assuming uniform thermal-ion density and temperature; we instead keep kinetic thermal-ion compressibility effects, in order to include the formation of a kinetic 
thermal-ion gap. Finally, a single toroidal mode number, $n=2$, is retained in the following simulations: this corresponds to neglecting fluid mode-mode coupling in the evolution of electromagnetic fields, while fully taking into account wave-particle nonlinearities.

A Tokamak equilibrium with shifted circular magnetic surfaces is considered, with aspect ratio $R_{0} / a=10$ and safety factor $q \approx q_{0}+\left(q_{a}-q_{0}\right)(r / a)^{2}$, with $q_{0}=1.9, q_{a}=2.3$ as shown in Fig. 1 (a). Thermal ions are characterised by isotropic Maxwellian initial distribution function with flat density and temperature profiles. The fast ("hot") ion (same species as the thermal ones) initial distribution function is instead an anisotropic Maxwellian in the form:

$$
F_{H} \propto \frac{n_{H}(\psi)}{T_{H}^{3 / 2}} \Xi\left(\alpha ; \alpha_{0}, \Delta\right) e^{-E / T_{H}} .
$$

Here, $E=\frac{1}{2} m_{H} U^{2}+M \Omega_{H}$ is the particle energy, $n_{H}(\psi)$ is the density profile, shown in Fig. 1 (b), $U$ is the parallel (to the equilibrium magnetic field) velocity, $M$ is the conserved magnetic momentum, $\alpha \equiv \cos ^{-1}\left(U / \sqrt{2 E / m_{H}}\right), \Omega_{H}=e_{H} B /\left(m_{H} c\right)$ is the cyclotron frequency, with $e_{H}$ and $m_{H}$ being charge and mass of fast ions, respectively, and $B$ is the magnitude of local equilibrium magnetic field. The quantity

$$
\Xi\left(\alpha ; \alpha_{0}, \Delta\right) \equiv \frac{4}{\Delta \sqrt{\pi}} \frac{\exp \left[-\left(\cos \alpha-\cos \alpha_{0}\right)^{2} / \Delta^{2}\right]}{\operatorname{erf}\left[\left(1-\cos \alpha_{0}\right) / \Delta\right]+\operatorname{erf}\left[\left(1+\cos \alpha_{0}\right) / \Delta\right]}
$$

models the anisotropy of the distribution function. Note that, in the isotropic limit $(\Xi=1)$, $T_{H}$ would assume the meaning of (uniform) temperature. In the present case, we assume an anisotropic initial distribution function characterised by parallel temperature much higher than the perpendicular one, by fixing $\Delta=0.1$ and $\alpha_{0}=\cos ^{-1}(1 / 2)$ or $\alpha_{0}=\cos ^{-1}(-1 / 2)$ for co-passing and counter-passing fast ions, respectively. These distribution functions are shown in Fig. 2(a) and (b), respectively. Other parameters are $v_{H} / v_{A 0}=0.3, v_{i} / v_{A 0}=0.06$, $\rho_{H 0} / a=0.01$ and $\rho_{i 0} / a=0.002$, with $\rho_{H} \equiv v_{H} / \Omega_{H}, \rho_{i} \equiv v_{i} / \Omega_{i}, \Omega_{i}$ being the thermal ion cyclotron frequency, $v_{A}$ the Alfvén velocity, and the subscript " 0 " corresponding to quantities computed at the magnetic axis. Note that the initial distribution function is not a proper equilibrium function, as it is not merely a function of constants of the unperturbed motion. For simplicity, we neglect the consequent initial relaxation of $F_{H}$ towards a genuine equilibrium, by suppressing the corresponding terms in the evolution of the perturbed distribution function $\delta F_{H}$; this does not affect our results in a significant way, as the typical particle orbit width (which causes $\psi$ not being a constant of the unperturbed motion) is few percent of the minor radius. In the following, for linear simulations, we shall consider a fraction of 
fast ions, $n_{H 0} / n_{i 0}$, ranging in the interval [0.0014,0.002]; for nonlinear simulations, we shall analyse only the weakest case, $n_{H 0} / n_{i 0}=0.0014$. In all the simulations, poloidal harmonics with $m$ from 1 to 6 will be retained.

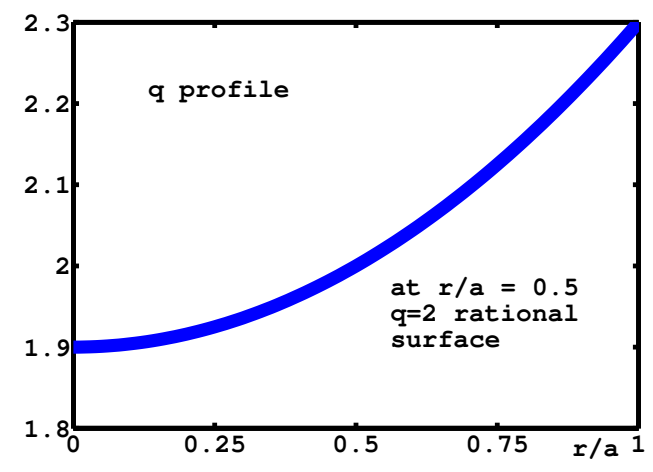

(a) Safety factor radial profile

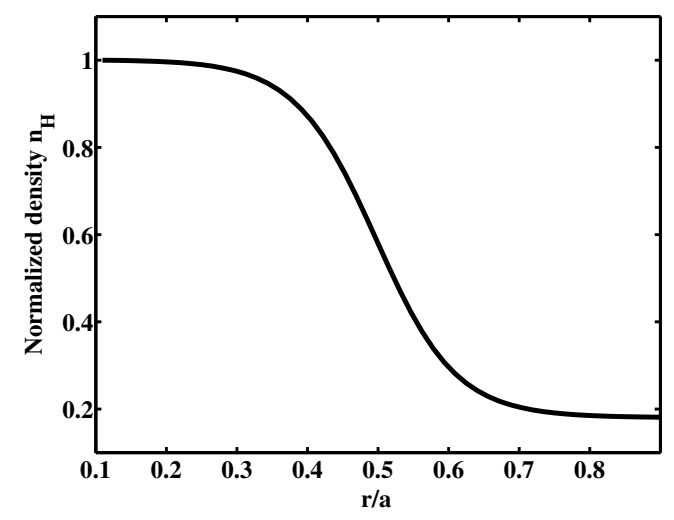

(b) EP density radial profile.

FIG. 1: The radial profile of safety factor (a) and normalized EP density radial profile $n_{H} / n_{H 0}(\mathrm{~b})$.

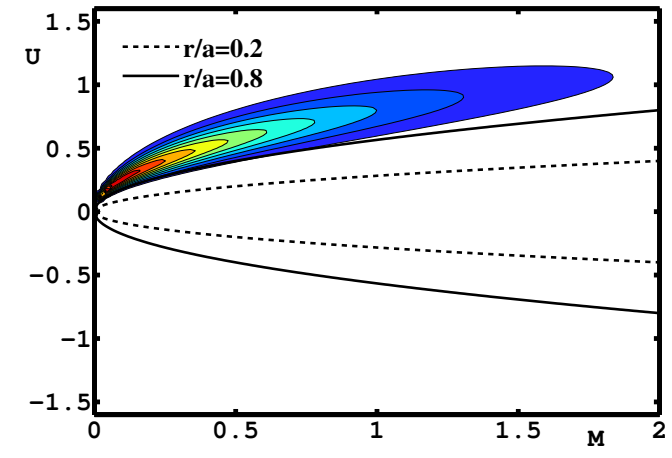

(a) Co-passing particles

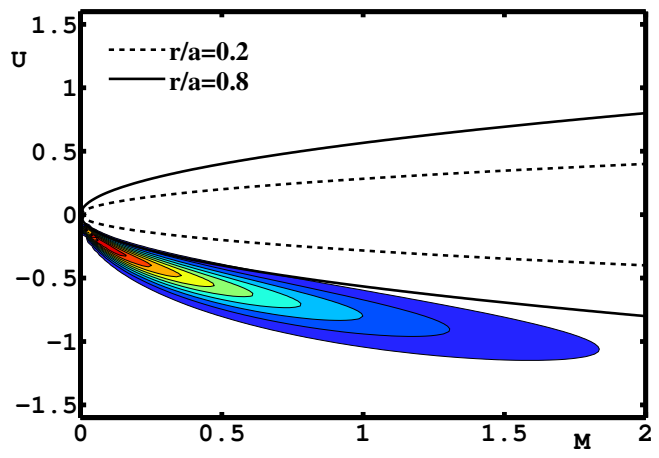

(b) Counter-passing particles

FIG. 2: Contour plot of the initial distribution function in the velocity space for co-passing (a) fast ions and counter-passing (b) fast ions. Note that, $M$ and $U$ are reported in units of $T_{H} / \Omega_{H 0}$ and $\sqrt{T_{H} / m_{H}}$. Passing-trapped boundaries evaluated at the inner $(r / a=0.2$, dashed) and outer $(r / a=0.8$, solid) limits of the mode's radial domain are shown. 


\section{LINEAR DYNAMICS}

\section{A. Overview of linear evolution}

In the absence of thermal-ion kinetic effects, the accumulation point on the shear Alfvén continuum at the rational surface would correspond to a frequency $\omega_{a c p}=0$. Once those effects are taken into account, the accumulation point moves to a finite frequency value. In this case, the radial dependence of the shear Alfvén continuum frequency has been computed, for the equilibrium here considered, by the linear gyrokinetic eigenvalue code LIGKA [47]. It is shown in Fig. 3 by the red curve; curves corresponding to $T_{e}=T_{i}$ (black) or the absence of kinetic thermal-ion effects (blue) are also reported, for comparison. When fastion drive is included, XHMGC simulations show the existence of an unstable BAE mode, with frequency in the kinetic thermal-ion gap, very close to the shear-Alfvén continuum accumulation point, as shown in Fig. 4. Both co-passing and counter-passing fast ions drive the mode unstable via transit resonance, with the same real frequency. The growth rates increase with increasing fast-ion fraction; those associated to counter-passing ions are approximately 10\% larger than those associated to co-passing ions, as shown in the Fig. 5 . The radial structure of different poloidal harmonics is shown in Fig. 6 for the co-passing and counter-passing ion cases: differences between the two cases are negligible.

In order to analyse wave-particle interactions in greater detail, we can look at the particleto-wave power transfer. Once averaged over poloidal $(\theta)$ and toroidal angle $(\phi)$, and weighted with the fast-ion distribution function, its density in the reduced phase space $(r, M, U)$ can be written as [19]:

$$
\begin{aligned}
P(r, M, U) & \equiv-2 \pi \int d \theta d \phi D_{z_{c} \rightarrow Z} F_{H} \frac{d E}{d t} \\
& \simeq \sum_{l} p_{l} \delta\left(r-r_{l}\right) \delta\left(M-M_{l}\right) \delta\left(U-U_{l}\right)
\end{aligned}
$$

where $D_{z_{c} \rightarrow Z}$ is the Jacobian of the transformation from canonical to guiding-center coordinates, $Z \equiv(r, \theta, \phi, M, U)$, and the latter expression for $P(r, M, U)$ represents its discrete form, as a sum over the simulation macroparticle population. In Fig. 7, the power transfer density $P(r, M, U)$, integrated over a radial shell centred around the mode peak, is shown for co-passing and counter-passing fast-ion cases. We observe that contour plots, in the two cases, are almost symmetric with respect to parallel velocity. Figure 8 shows the similarity 
of the two cases subsists also with respect to the radial profile of the power transfer density integrated over the whole velocity space.

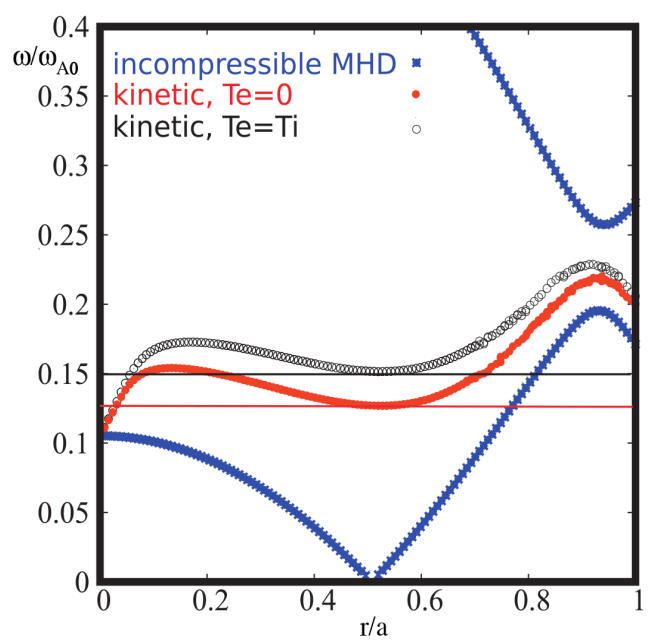

FIG. 3: Shear Alfvén continuum for the equilibrium considered in the present paper, calculated by LIGKA (red curve). The same continuum for $T_{e}=T_{i}\left(\right.$ instead of $\left.T_{e}=0\right)$ and in the absence of thermal ion effects are represented by black and blue curves, respectively. The accumulation-point frequencies for the former two cases are indicated by red $\left(\omega=0.125 \omega_{A 0}\right)$ and black $\left(\omega=0.15 \omega_{A 0}\right)$ lines, respectively, with $\omega_{A 0} \equiv v_{A 0} / R_{0}$ being the on-axis Alfvén frequency. 


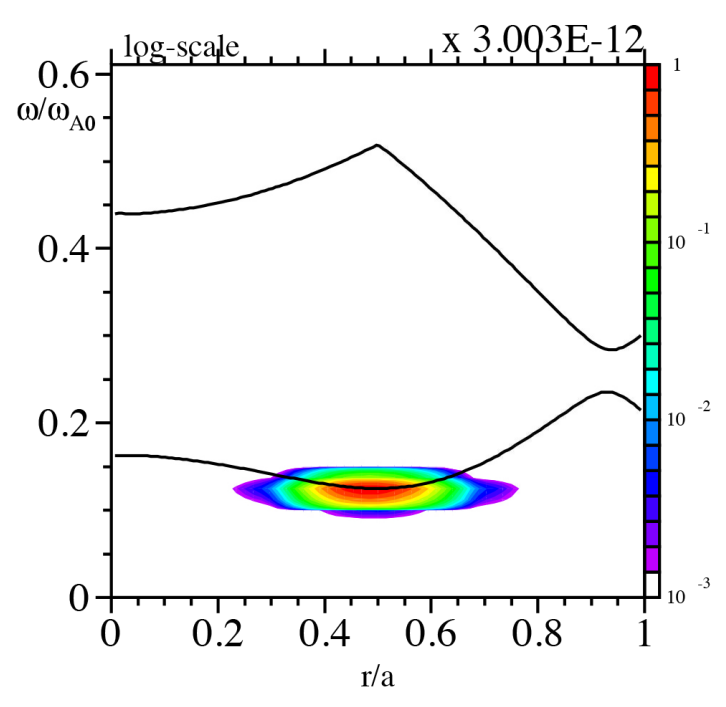

(a) co-passing particles

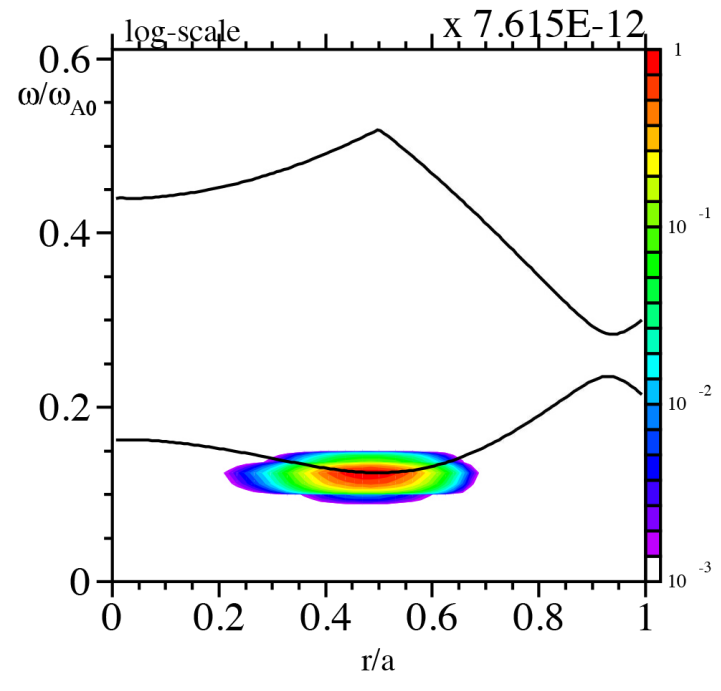

(b) counter-passing particles

FIG. 4: Energy spectrum for the scalar potential, in the $(r, \omega)$ plane, for the BAE driven by fast ions with $n_{H 0} / n_{i 0}=0.0014$. The solid line represents the shear Alfvén continuum with thermal-ion kinetic effects included. The mode is located in the thermal-ion gap. 


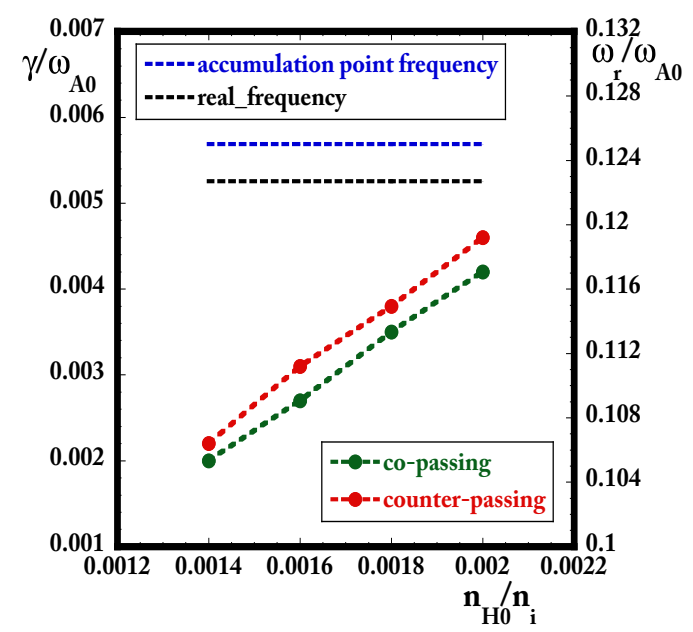

FIG. 5: Real frequency and growth rate for BAEs driven by co-passing and counter-passing fast ions. at different fast-ion densities. No differences are observed, between co-passing and counter-passing cases, with respect to the real frequency (black dashed curve); the accumulation-point frequency is also plotted (blue dashed curve). The growth rate increases with increasing fast-ion density, with a threshold for instability of order $n_{H 0} / n_{i 0} \sim 0.001$. The counter-passing fast-ion case (red curve) is approximately $10 \%$ more unstable than the co-passing one (green curve).

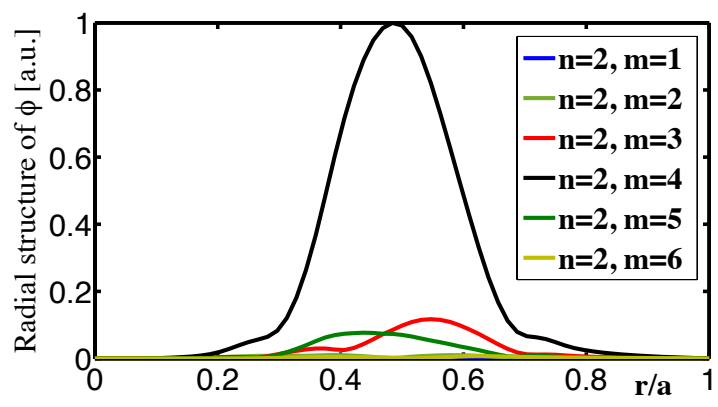

(a) co-passing particles

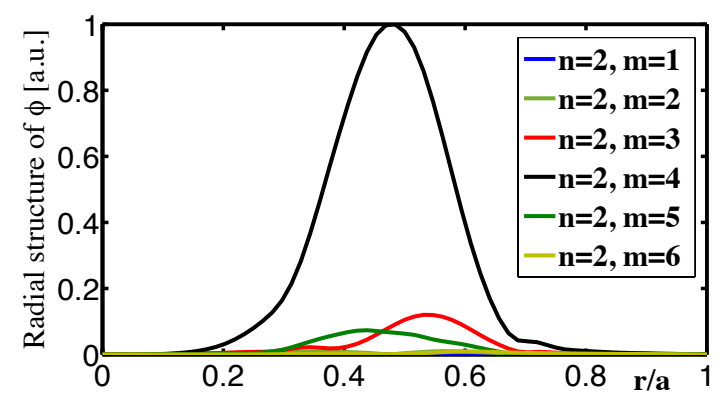

(b) counter-passing particles

FIG. 6: Radial structure of the different poloidal harmonics for the co-passing fast-ion case (a) and the counter-passing one (b). 


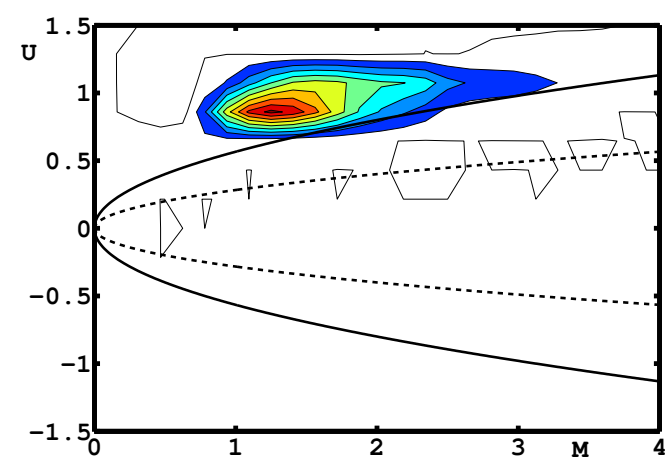

(a) co-passing particles

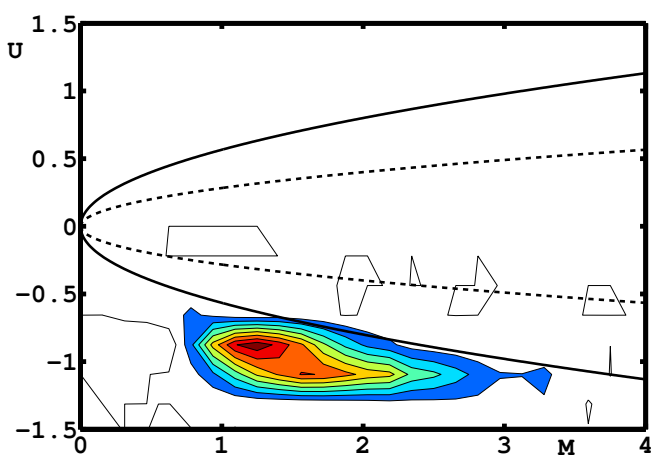

(b) counter-passing particles

FIG. 7: Power transfer density $P(r, M, U)$, integrated over a radial shell centred around the mode peak, for co-passing (a) and counter-passing (b) fast-ion cases. $M$ and $U$ normalisation are the same as in Fig. 2. Same conventions as in Fig. 2 is also used for dashed and solid lines.

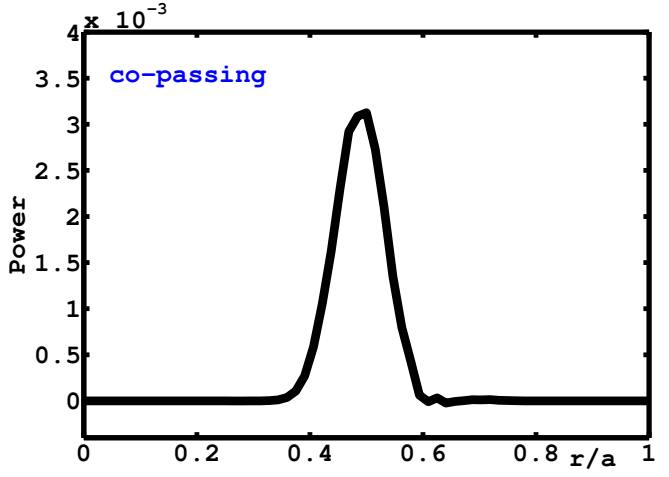

(a) co-passing particles

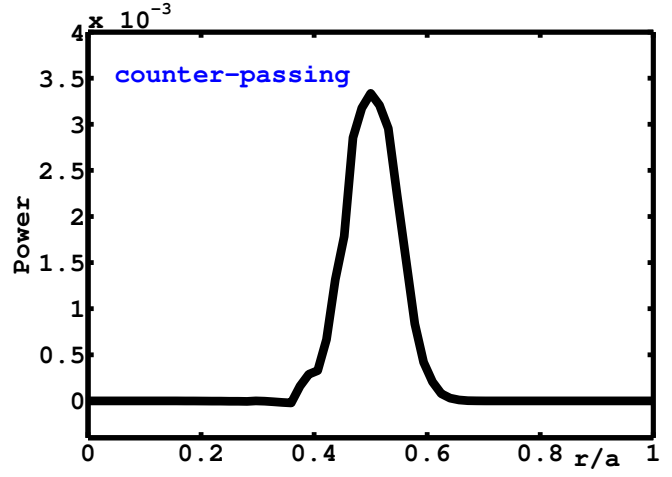

(b) counter-passing particles

FIG. 8: Radial profile of the linear-phase power transfer density $P(r, M, U)$ integrated over the whole velocity space, for co-passing (a) and counter-passing (b) fast-ion cases.

\section{B. Resonance analysis}

Differences between the two cases become evident only in a detailed analysis of the resonant particle behaviour. Following Ref. [19], we observe that, in the presence of an electromagnetic field perturbation characterised by a single toroidal number and constant frequency, the quantity $C \equiv \omega P_{\phi}-n E$, with $P_{\phi}$ being the toroidal angular momentum, is a 
constant of the (perturbed) motion. At the leading order, $P_{\phi} \simeq m_{H} R U+e_{H} R_{0}\left(\psi_{\text {eq }}-\psi_{\text {eq0 }}\right) / c$, with $\psi_{\text {eq }}$ defined by $\mathbf{B} \equiv R_{0} B_{\phi 0} \nabla \phi+R_{0} \nabla \psi_{\text {eq }} \times \nabla \phi$. Then, if we cut the phase space into "slices" of given constant of motions $M$ and $C$, particles remain in the same slice during the whole evolution of the system. The wave-particle power exchanges within different slices are then independent of each other, and the analysis of a single-slice behaviour gives us a microscopic view of the mode-particle dynamics, possibly hidden to a macroscopic (that is, integrated) inspection.

In order to explore dynamics within a single slice (and, in particular, the characteristic particle frequencies; namely, bounce/transit frequency $\omega_{b}$ and precession frequency $\omega_{D}$ ), we sample that slice by means of a set of test particles, evolved in the self-consistently simulated fields. The selection of test particles is discussed in detail in Ref. [19]. Once a significant couple, $\left(M_{0}, C_{0}\right)$, is identified, corresponding to a power-transfer peak in the linear phase, we initialise a set of test particles with $M=M_{0}, C=C_{0}$ and $\theta=0$ (that is, on the equatorial plane). Each particle has a different radial coordinate $r$ and the corresponding value of $U=U\left(r, \theta=0, M_{0}, C_{0}\right)$. Such a set is then replicated at different values of the toroidal angle $\phi$.

For each particle, we can compute $\omega_{b}$ and $\omega_{D}$ by collecting particle information every time the particle crosses the equatorial plane $(\theta=0)$ at its outmost $R$ position (in the following we shall use the attribute "equatorial-plane" to refer to such condition). The resonance frequency is given, as a combination of these characteristic frequencies, by

$$
\omega_{\mathrm{res}} \equiv n \omega_{D}+[(n \bar{q}-m) \sigma+k] \omega_{b}
$$

Here [20],

$$
\begin{gathered}
\omega_{D} \equiv\left[\frac{\Delta \phi}{2 \pi}-\sigma \bar{q}\right] \omega_{b}, \\
\sigma=\operatorname{sgn}(U), \\
\omega_{b} \equiv \frac{2 \pi}{\tau_{b}},
\end{gathered}
$$

$\Delta \phi$ is the change in toroidal angle over the bounce time $\tau_{b}$ (the time needed to complete a poloidal orbit), defined as

$$
\tau_{b} \equiv \oint \frac{d \theta}{\dot{\theta}}
$$

and $\bar{q}$ is the safety factor integrated along the particle orbit:

$$
\bar{q} \equiv \frac{\sigma}{2 \pi} \oint q d \theta
$$


The resonance frequency can be represented, for the set of test particles, as a function of the equatorial-plane radius only. Figure 9 reports $\omega_{\text {res }}(r)$ for different values of the bounce harmonic $k$, along with the effective mode frequency. It can be seen that the relevant bounce harmonic for both co-passing and counter-passing particles is $k=1$. The various ingredients entering the resonance frequency for co-passing and counter-passing test-particles are shown in Fig. 10. Both the bounce frequency, $\omega_{b}$, and the factor $(n \bar{q}-m) \omega_{b}$ have similar profiles for the two test-particle sets. The precession frequency, $\omega_{D}$, is negligible in both cases. The opposite sign of the parallel velocity, $\sigma$, however, makes the $\omega_{\text {res }}$ profiles very different; in particular, co-passing test particles have a much flatter profile, especially in the inner radial region.

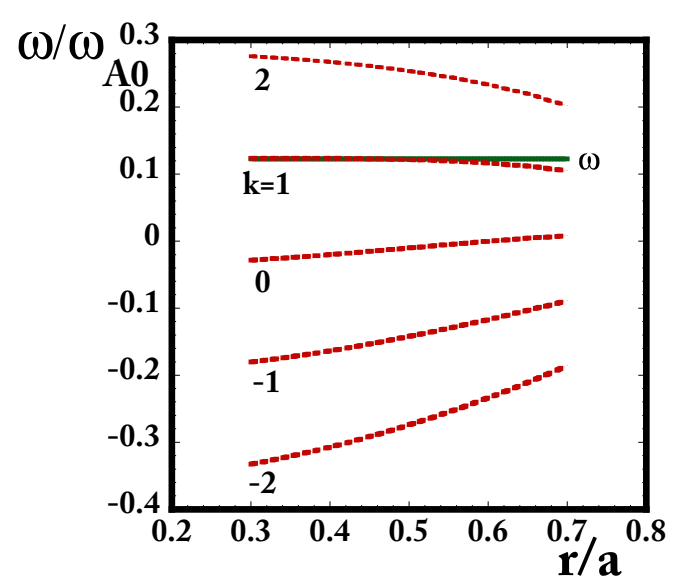

(a) co-passing particles

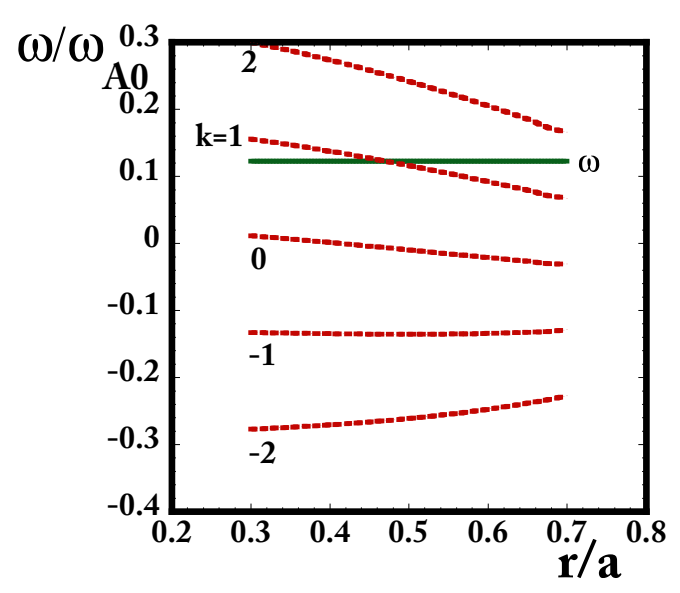

(b) counter-passing particles

FIG. 9: Resonance frequency, $\omega_{r e s}\left(r, M_{0}, C_{0}, k\right)$ for co-passing (a) and counter-passing (b) fast ions, for different values of the bounce harmonic $k$. The relevant bounce harmonic for both co-passing particles and counter-passing particles is $k=1$. 


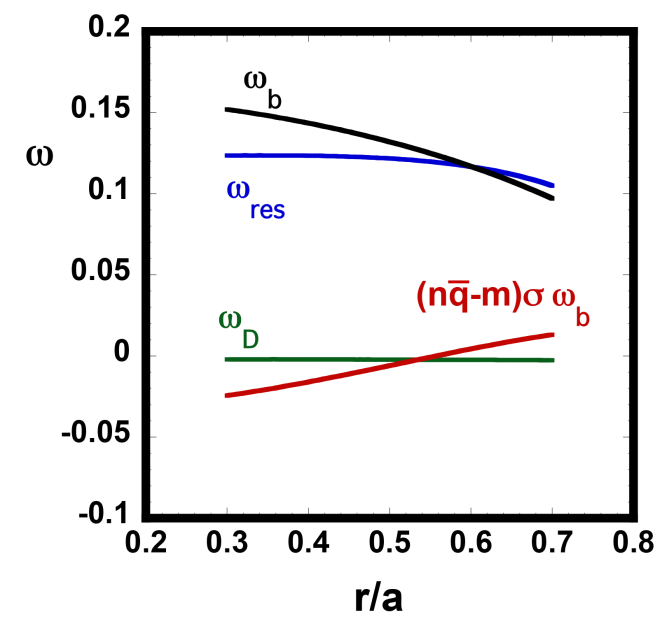

(a) co-passing particles

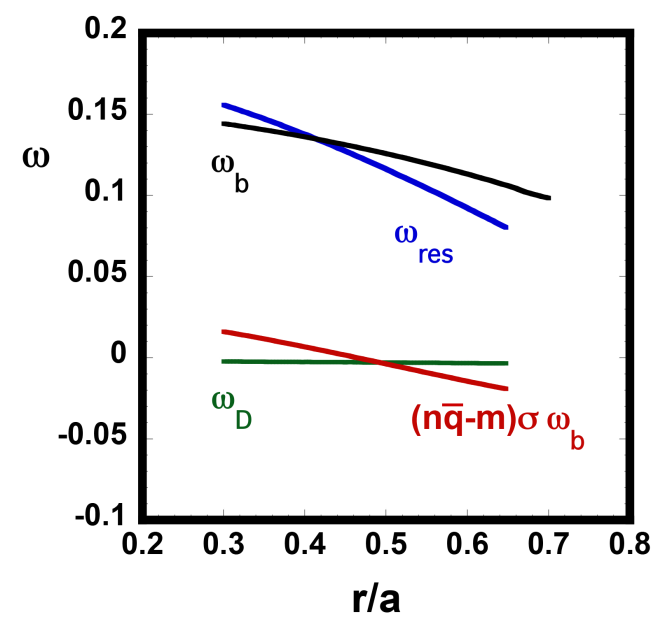

(b) counter-passing particles

FIG. 10: The various ingredients entering the resonance frequency, $\omega_{\text {res }}$, for co-passing (a) and counter-passing (b) test ions. Though both the bounce $\left(\omega_{b}\right)$ and the precession $\left(\omega_{D}\right)$ frequency profile, as well as the radial profile of the factor $(n \bar{q}-m) \omega_{b}$, are similar for both sets, the opposite sign of parallel velocity, $\sigma$, make the $\omega_{\text {res }}$ profile for co-passing ions much flatter than for counter-passing ones.

The resonance condition $\omega-\omega_{\text {res }}\left(r, M_{0}, C_{0}, k\right)=0$ identifies an equatorial-plane radial position $r=r_{\text {res }}\left(\omega, M_{0}, C_{0}, k\right)$ where the resonance takes place. If the mode is characterized, in the linear phase, by a growth rate $\gamma$, the resonance will be anyway significant in a radial layer around $r_{\text {res }}$, of width $\Delta r_{\text {res }}$, approximately defined by the following condition:

$$
\left|\omega-\omega_{\text {res }}\left(r, M_{0}, C_{0}, k\right)\right| \lesssim \gamma
$$

The resonance width is larger for larger growth rates and/or weaker radial dependence of $\omega_{\text {res. }}$ In Fig. 11, the determination of the layer is reported for co-passing and counter-passing ions. In the considered case, the two species behave differently due to the different sign of their velocity, which yields a different radial dependence of the resonance frequency: copassing ions are characterised by a large resonance width, because of the flat radial profile of $\omega_{\text {res }}$; counter-passing ions have instead a narrow resonance, due to the much steeper $\omega_{\text {res }}$ profile. In Sect. IV, we will show that this difference causes the different nonlinear evolution of the mode amplitude in the two cases. 


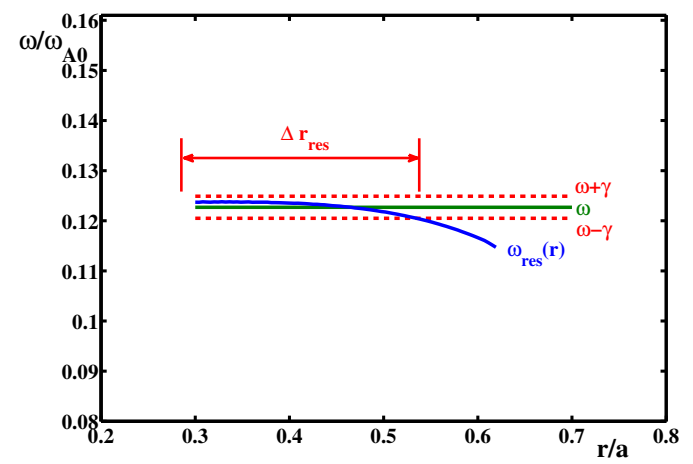

(a) co-passing particles

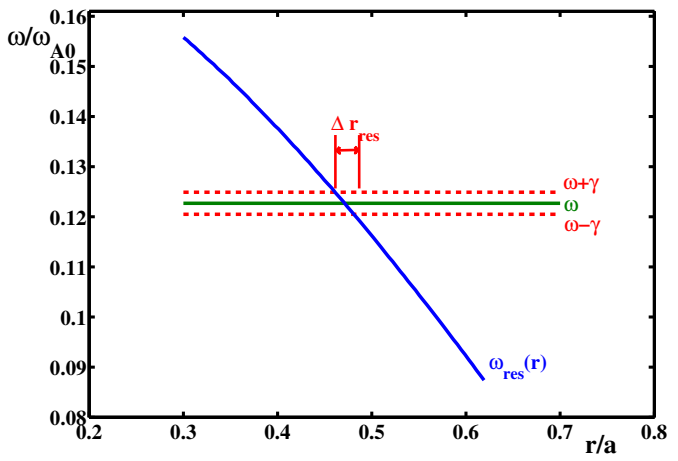

(b) counter-passing particles

FIG. 11: Resonant layers for the specific test-particle sets selected for co-passing (a) and counter-passing (b) fast ions. The resonance width is estimated here as the width of the radial interval for which $\left|\omega-\omega_{\text {res }}\left(r, M_{0}, C_{0}, k\right)\right| \leq \gamma$. It is much larger for co-passing ions than counter-passing ones.

The radial density of power transfer, for the considered slice, can be qualitatively represented by the following symbolic form [19]:

$$
P\left(r, M_{0}, C_{0}\right) \sim\left\langle\frac{D_{z_{c} \rightarrow \hat{Z}}}{\left|\omega+i \gamma-\omega_{\text {res }}\right|}\left[\left(\frac{d \hat{Z}^{i}}{d t}\right)_{1} \frac{\partial F_{H}}{\partial \hat{Z}^{i}}\right]\left[\left(\frac{d \hat{Z}^{j}}{d t}\right)_{1} \frac{\partial E}{\partial \hat{Z}^{j}}\right]\right\rangle .
$$

Here, we have adopted a coordinate system $\hat{Z} \equiv(r, \theta, \phi, M, C)$, and the subscript " 1 " stands for the perturbed part of the phase-space velocities, proportional to the perturbed fields. The average is performed by integrating each test particle contribution along the last poloidal orbit (that connecting two successive equatorial-plane crossings) and summing the results over the class of test particles characterised, on the equatorial plane, by the same value of $r$ and different values of $\phi$. This simplified form makes clear that the effective power exchange structure is determined not only by the resonance condition (Eq. 10), but also by the spatial mode structure and the distribution-function gradients computed in the resonant region. Note that, once the coordinate system $\hat{Z}$ is adopted and provided the conditions for $C$ conservation are satisfied, gradients with respect to $M$ and $C$ do not enter the power exchange, as the corresponding phase-space velocities are zero. Then, the main free-energy source for instability comes, at the lowest order, from the radial derivative of $F_{H}$. Looking at the two factors directly related to the existence of a perturbed field (mode structure and resonant layer), we see, from Fig. 12, that the co-passing ion case and the counter-passing 
ion case falls into two different limits. For co-passing ions, the resonance width is larger than the mode width, which then yields the most restrictive constraint to the power transfer radial extension. On the contrary, for counter-passing ions, the main constraint comes from the resonance width (smaller than the mode width). A more stringent limitation could be set by the width of the region where the radial gradient of the initial distribution function for $M=M_{0}$ and $C=C_{0}$ is relevant, if such width is smaller than both mode and resonance ones. This is not the case, however, in the considered scenario: the gradient width comes out to be slightly larger than the mode width. In Sect. IV], we will analyse how the lineardynamics features described in the present Section determine the nonlinear evolution of modes in the co-passing and counter-passing ion cases.

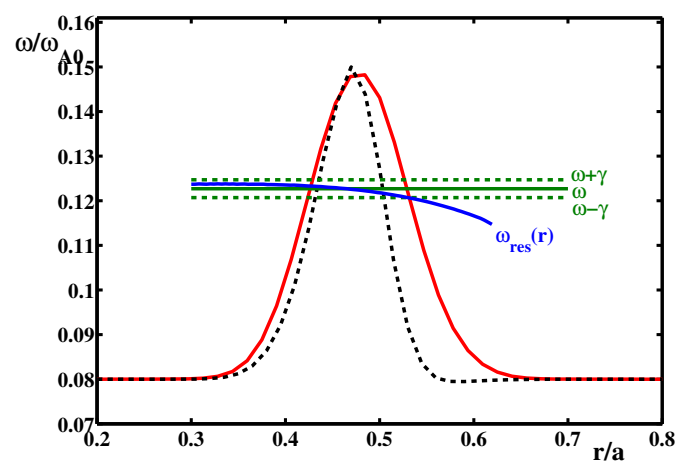

(a) co-passing particles

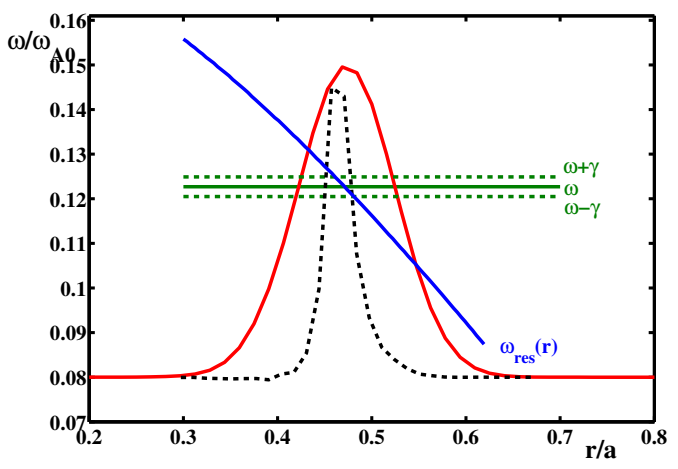

(b) counter-passing particles

FIG. 12: Resonance radial structure compared with the mode structure (red solid curve) and the effective power transfer one (black dashed curve), for the co-passing (a) and counterpassing (b) fast-ion selected sets. The radial extension of power transfer density is limited by mode radial width for co-passing ions; by resonance width, for counter-passing ions.

\section{NONLINEAR DYNAMICS}

Figure 13 shows the nonlinear evolution of the perturbed scalar potential amplitude, in the weakest case reported in Fig. $5\left(n_{H} / n_{i}=0.0014\right)$, for both co-passing and counterpassing fast-ion drive. Saturation is reached at a larger amplitude in the co-passing fastion case than the counter-passing one, in spite of a smaller growth rate. Looking only at macroscopic mode and particle linear-dynamics features, such as mode frequency (Fig. 4) 
and structure (Fig. 6) or radial profile of the integrated (over the velocity space) power transfer (Fig. 8), very similar in the two cases, this result could seem counterintuitive; also because the transition from linear to saturation phase is not accompanied, in any of the two cases, by significant modification of mode frequency (Fig. 14) and structure (Fig. 15) or fast-ion density profile (Fig. 16). We have already seen, however, in Sect. III B, that the phase space can be discretised into slices of constant $M$ and $C$, with particles belonging to each slice being conserved. The integrated mode-particle power exchange can then be seen as the collective effect of the different slices, each of them evolving in an independent way. The analysis of a single slice corresponding to a peak of resonant drive (in the following, a "resonant slice") has shown that the two cases deeply differ with respect to the radial profile of the resonance frequency. This causes the radial extension of the power transfer to be limited by the mode width, in the co-passing ion case; by the resonance width, in the counter-passing ion case.

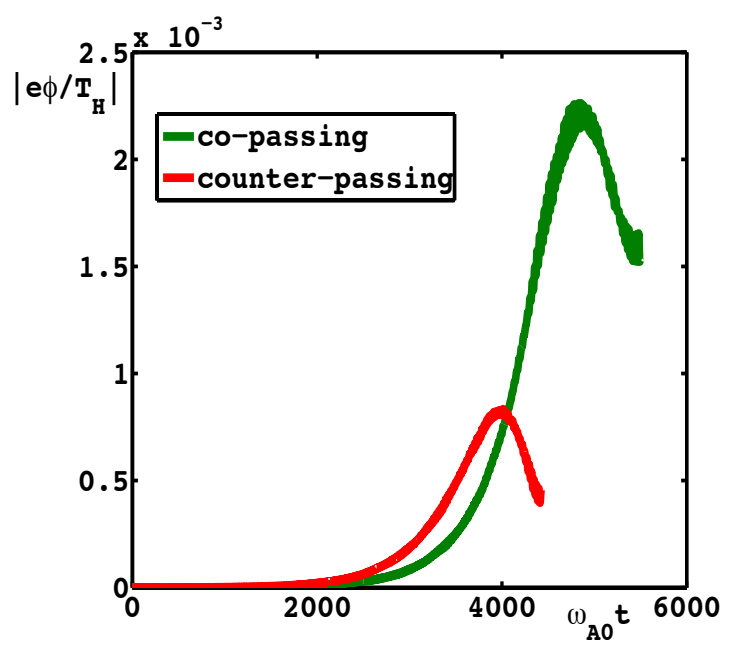

FIG. 13: Time evolution of the normalised scalar potential amplitude $\left|e \phi / T_{H}\right|$ at the peak of the mode structure. 


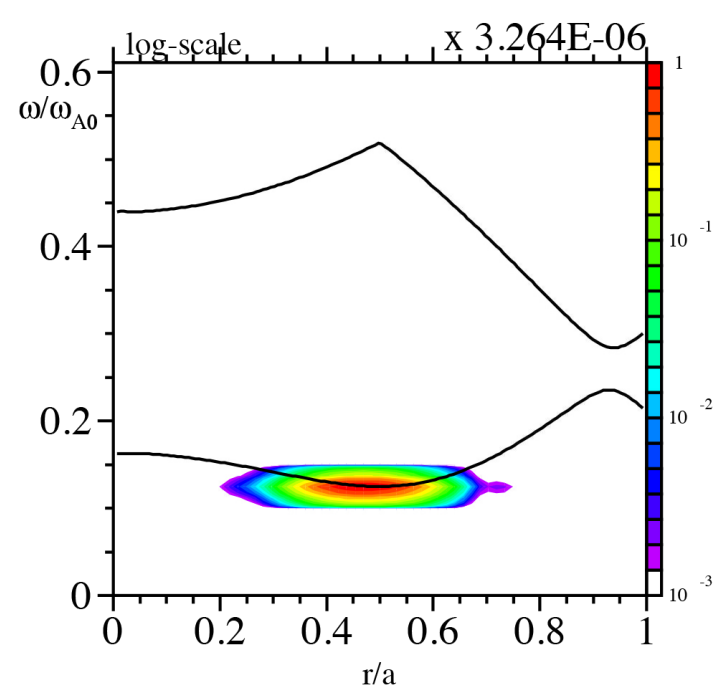

(a) co-passing particles

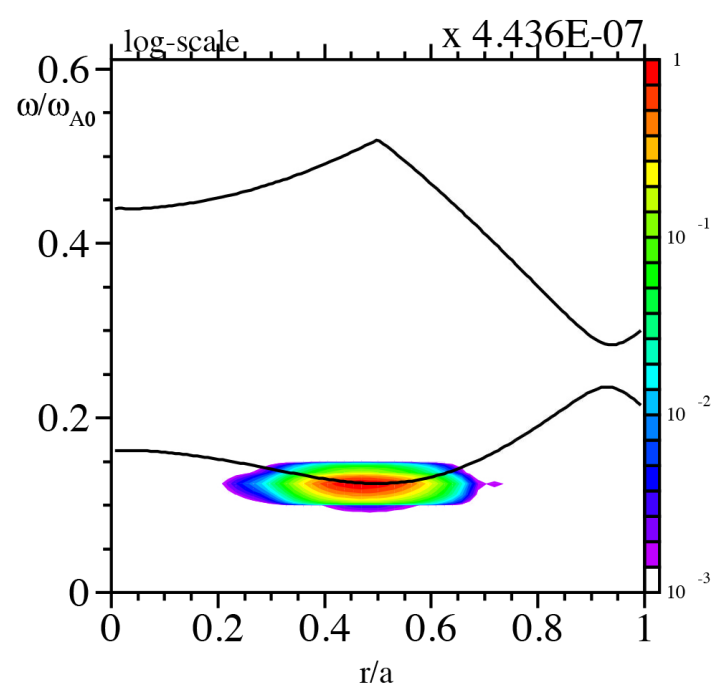

(b) counter-passing particles

FIG. 14: Mode frequency at the saturation, for the co-passing (a) and counter-passing (b) fast-ion cases.

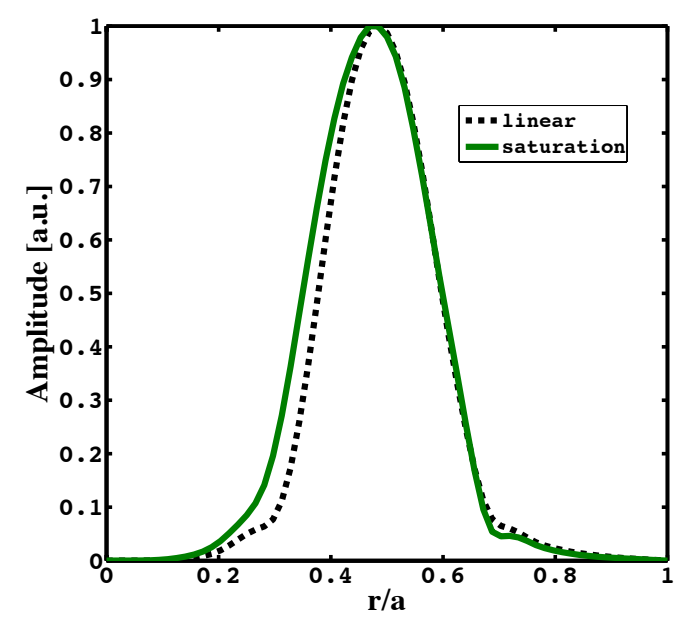

(a) co-passing particles

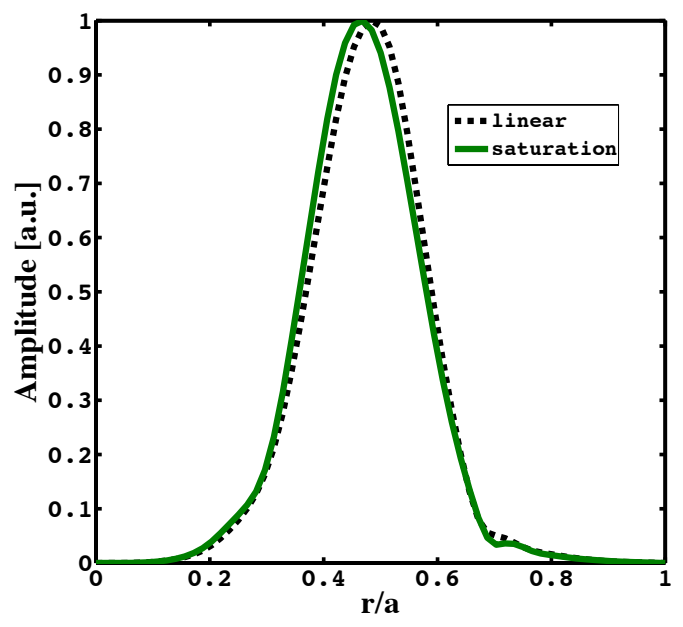

(b) counter-passing particles

FIG. 15: Comparison between linear and saturation mode radial structures.

In Ref. [19] it has been shown that following the evolution of test particles belonging to a resonant slice, by means of Hamiltonian mapping techniques, allows for identifying the mode saturation mechanism (cf. the Appendix for a short description of the method). 
In particular, it has been described the formation of topologically closed particle orbits in the plane $\left(\Theta, P_{\phi}\right)$ (with $\Theta$ being the wave-particle phase) around the resonance condition, $P_{\phi}=P_{\phi \text { res }}$, because of the mode-particle interaction. The consequent mixing of particles originating from the higher-density side of the resonance with particles originating from the lower-density one produces density profile distortions. In the following, we shall indicate such process as "density flattening", although it can even consist in a density-profile inversion and it is accompanied by a density steepening at the boundaries of the interested region.

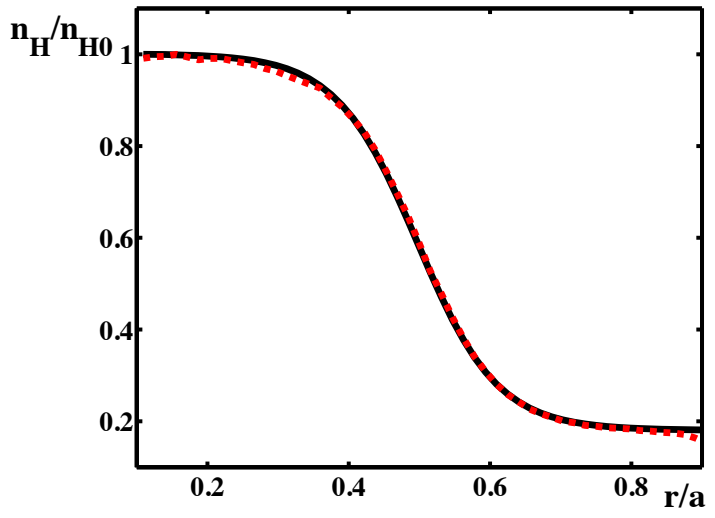

(a) co-passing particles

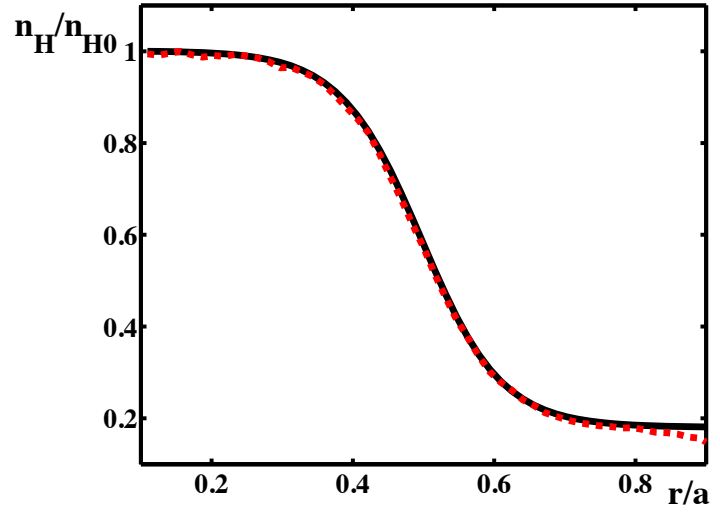

(b) counter-passing particles

FIG. 16: Fast ion density radial profile in the linear phase (black curve) and at saturation (red curve), for the co-passing (a) and counter-passing (b) fast-ion cases.

Saturation is reached when the density flattening covers the whole effective power-transfer region, determined by the coexistence of mode strength and frequency matching. Note that this would be true even in a case (different from the scenario considered in this paper) in which the power transfer region in the linear phase were limited by the finite radial extension of an equilibrium fast ion density gradient narrower than mode and resonance width. Indeed, in such case, the large gradients built by the density profile evolution on both sides of the flattening region would be able to yield the free-energy source for driving the mode even in where, in the linear phase, the gradient was negligible. In other words, the original narrowness of the gradient region would not play any role in limiting the evolution of the density profile: such evolution would eventually stop only when facing the bounds posed by mode or resonance width.

Figure 17 compares the test-particle density profile at saturation with the unperturbed 
one. It can be seen that the width of the density-flattening region is much larger for copassing ions than for counter-passing ones. This is consistent with the fact that, in the co-passing ion case, the power-transfer region is limited by the mode width; for the counterpassing ions, it is limited by the (smaller) resonance width. Figure 18 reports a comparison between the radial width of resonance, mode structure and density-flattening region for copassing and counter-passing ions, at different values of the linear growth rate. In the former case, the width of the saturation flattening region is limited by the mode width. In the latter case, it is limited by the resonance width in the low- $\gamma$ limit; by the mode width, in the large- $\gamma$ limit.

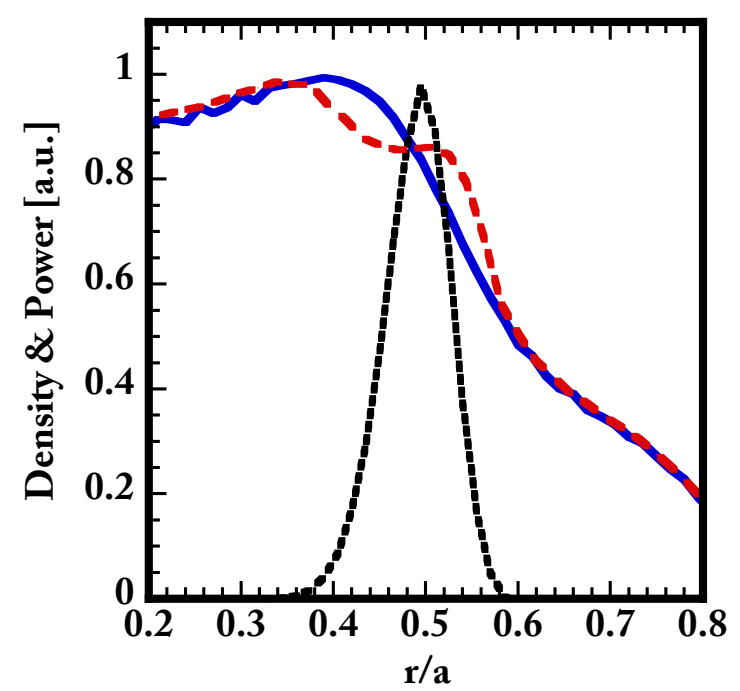

(a) co-passing particles

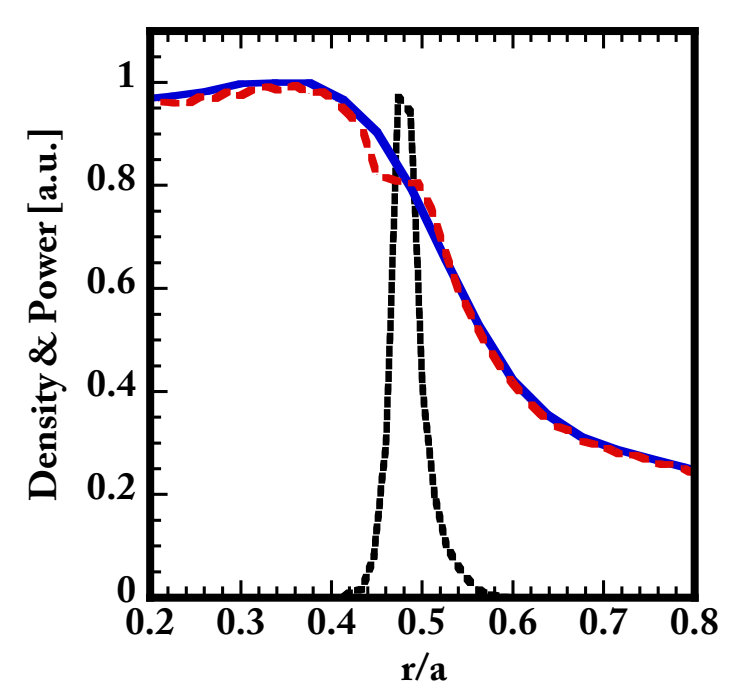

(b) counter-passing particles

FIG. 17: Test-particle density profile at saturation (red curve) for the test-particle samples compared with the unperturbed one (blue curve). The width of the density flattening region at saturation, which, for both cases, extends over the whole radial region of power exchange (black dashed line), is much larger for co-passing fast ions (left) than for counter-passing ones (right). 


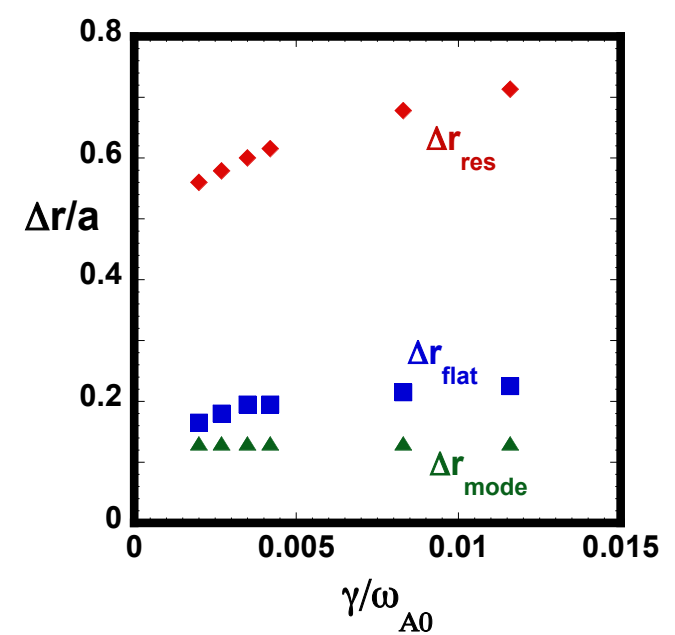

(a) co-passing particles

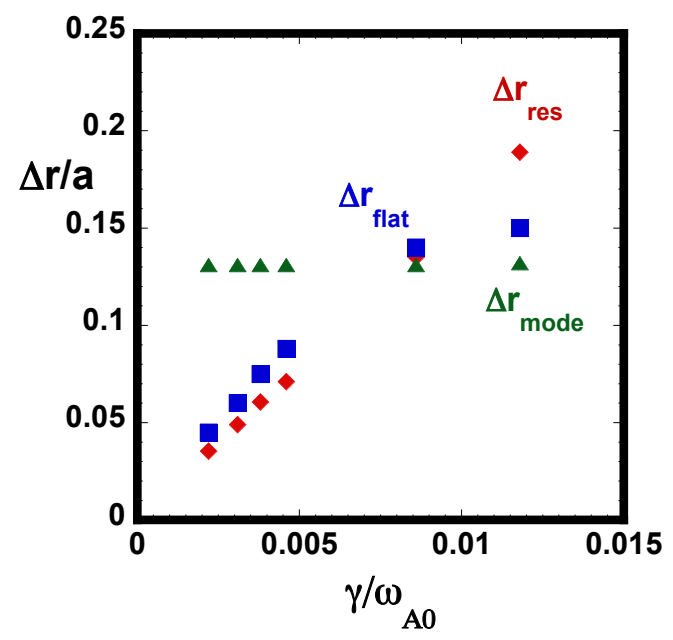

(b) counter-passing particles

FIG. 18: Radial width of mode, resonance and density-flattening region (at saturation), at different values of the linear growth rate, for co-passing (left) and counter-passing (right) fast ions. In the former case, the width of the saturation flattening region is limited by the mode width. In the latter case, it is limited by the resonance width in the low- $\gamma$ limit; by the mode width, in the large- $\gamma$ limit.

Figure 19 shows the saturation field amplitude obtained, for co-passing and counterpassing fast ions, at different fast-ion densities, versus the linear growth rate of the mode. We observe that, in the low growth rate limit, the amplitude values reached in the co-passing ion cases are larger than those in the counter-passing cases, and the scaling with the growth rate is weaker in the former than in the latter cases (the amplitude ratio then reduces with increasing $\gamma$ ). In the large growth rate limit, the amplitude values converge towards the same values and to the same weaker $\gamma$ dependence. 


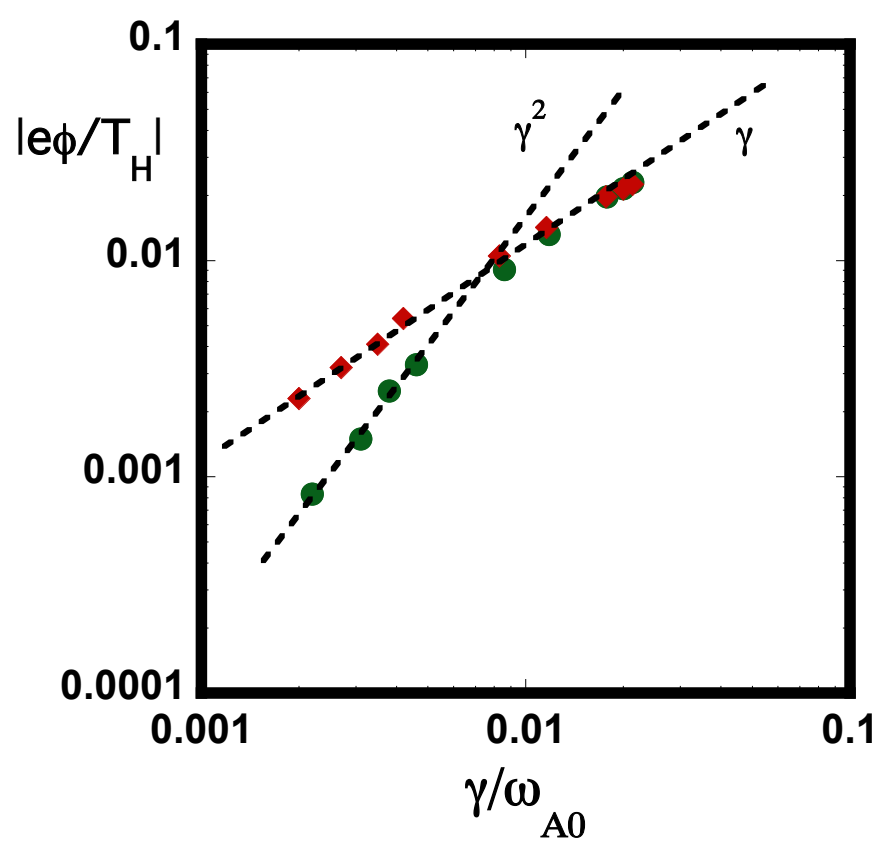

FIG. 19: Scaling of saturation amplitude of scalar potential versus $\gamma$ for co-passing particles (diamonds) and counter-passing particles (circles). The reference quadratic and linear $\gamma$ scaling are also shown. Note that both coordinates are reported in logarithmic scale.

\section{HEURISTIC MODEL}

We can resort to a simplified model to interpret the results presented in Fig. 19. We have seen that the basic saturation mechanism is resonant-particle density distortion around the resonance radius (cf. Figure 17), which is conventionally indicated here as density flattening. The radial extension of the region involved in this process is of the same order of that of the structures formed in the $\left(P_{\phi}, \Theta\right)$ plane (cf. the Appendix and Fig. 21 therein). Such extension can be evaluated by describing particle orbits in the corresponding $(r, \Theta)$ plane with the following nonlinear pendulum system:

$$
\begin{aligned}
\frac{d x}{d t} & \equiv-A \frac{S}{|S|} \sin \Theta \\
\frac{d \Theta}{d t} & \equiv S x .
\end{aligned}
$$

Here, $x \equiv r-r_{\text {res }}$ is the radial distance from the resonant surface (where $d \Theta / d t=0$ ), $A>0$ is proportional to the mode amplitude (responsible for the radial drift, Eq, 12), and 
$S$ is related to the radial slope of the resonance frequency (cf. the blue curve in Fig 11): $\omega_{\text {res }} \simeq \omega-S\left(r-r_{\text {res }}\right)$, which is responsible for the wave-particle phase change in Eq. 13 . Let us first assume that the growth rate of the mode is sufficiently small that we may treat the mode amplitude as a constant. We can evaluate the radial extension of the closed orbit structure by the radial width of the separatrix between closed and drifting orbits. To this aim, we note that the quantity

$$
E(x, \Theta) \equiv \frac{1}{2}\left(\frac{d \Theta}{d t}\right)^{2}-A|S| \cos \Theta=\frac{1}{2}(S x)^{2}-A|S| \cos \Theta
$$

is a constant of motion. The separatrix crosses the axis $x=0$ at $\Theta=\pi$. Then, it is characterised by an $E$ value

$$
E_{\mathrm{sep}} \equiv E(0, \pi)=A|S| .
$$

We can then compute its half width $x_{\text {sep }}$ from the condition

$$
E\left(x_{\mathrm{sep}}, 0\right)=E_{\mathrm{sep}},
$$

that is

$$
\frac{1}{2}\left(S x_{\mathrm{sep}}\right)^{2}-A|S|=A|S|
$$

from which

$$
\left|x_{\mathrm{sep}}\right|=2\left(\frac{A}{|S|}\right)^{1 / 2} .
$$

Identifying the radial width of the density flattening region with the full width of the separatrix,

$$
\Delta r_{\text {flat }} \simeq 2\left|x_{\text {sep }}\right|,
$$

we can assume that saturation occurs when

$$
\Delta r_{\text {flat }} \simeq \min \left[\Delta r_{\text {mode }}, \Delta r_{\text {res }}\right]
$$

i.e., the minimum between mode and resonance width. Consistent with the linearisation of $\omega_{\text {res }}$ around $r_{\text {res }}, \Delta r_{\text {res }}$ can be evaluated as

$$
\Delta r_{\mathrm{res}} \simeq 2 \frac{\gamma}{|S|}
$$

Let us now refer to the two different cases shown in Fig. 12, and adopt the superscripts "+" for the co-passing ion case, "-" for the counter-passing ion one. Let us also neglect the 
differences between the two cases in terms of mode structure and growth rate: we will focus on the different radial dependence of resonance frequencies (that is, on the different slopes $S^{+}$and $S^{-}$). From Fig. 18, we see that saturation conditions can be written, respectively, as

$$
\Delta r_{\text {flat }}^{+} \simeq \Delta r_{\text {mode }}
$$

or

$$
A^{+}=\frac{1}{16}\left|S^{+}\right| \Delta r_{\text {mode }}^{2}
$$

and

$$
\begin{array}{ll}
\Delta r_{\text {flat }}^{-} \simeq \Delta r_{\text {res }}^{-} & \text {for low } \gamma \\
\Delta r_{\text {flat }}^{-} \simeq \Delta r_{\text {mode }} & \text { for large } \gamma
\end{array}
$$

or

$$
\begin{array}{ll}
A^{-}=\frac{1}{4} \frac{\gamma^{2}}{\left|S^{-}\right|} & \text {for low } \gamma \\
A^{-}=\frac{1}{16}\left|S^{-}\right| \Delta r_{\text {mode }}^{2} & \text { for large } \gamma
\end{array}
$$

Following Refs. 19] and [20], we call "radial decoupling" the saturation mechanism in the co-passing ion case, as well as in the large- $\gamma$ limit for counter-passing ions, and "resonance detuning" that in the low- $\gamma$ counter-passing case. It can be seen that, even this simple model is able to capture the quadratic $\gamma$ scaling observed in the resonance detuning regime, and the weaker dependence typical of the radial decoupling one; it fails, however in justifying the linear character of such weaker dependence.

We can improve the model by taking into account that treating the mode amplitude $A$ as a constant, while evaluating its effect on the density flattening, is appropriate only in the very low growth rate limit: $\gamma T_{\text {orb }} \ll 1$, with $T_{\text {orb }}$ being the time needed for a particle to complete its closed orbit. Let us introduce the equations for the time evolution of $A$ in the following form:

$$
\frac{d A}{d t}=\bar{\gamma} A
$$

with

$$
\bar{\gamma}=\gamma\left\{1-\frac{\Delta r_{\text {flat }}}{\min \left[\Delta r_{\text {mode }}, \Delta r_{\text {res }}\right]} \cdot\right\}
$$

Equations 28 and 29 have been implicitely used, in the previous derivation, defining the saturation condition as $\bar{\gamma}=0$ and assuming the flattening region width given by Eq. 19 . 
For a given (static) amplitude $A, T_{\text {orb }}$ is an increasing function of $\left.\Theta_{0} \in\right] 0, \pi\left[\right.$, where $\Theta_{0}$ is the value of $\Theta$ at which the orbit crosses the axis $x=0$ :

$$
T_{\text {orb }}\left(\Theta_{0}\right)=\frac{4}{(|S| A)^{1 / 2}} K\left(\sin ^{2} \frac{\Theta_{0}}{2}\right)
$$

with $K$ being the elliptic integral of the first kind [48]. Then, deeply wave-trapped particles $\left(\Theta_{0} \rightarrow 0\right)$ will be characterised by a smaller $T_{\text {orb }}$ than particles close to the separatrix $\left(\Theta_{0} \rightarrow\right.$ $\pi$ ). We can then modify the estimate of $\Delta r_{\text {flat }}$ given in Eq. 19 by assuming that the density flattening involves, at a certain time, only a fraction of the whole region contained within the virtual separatrix that would correspond, in a static limit, to the actual instantaneous amplitude. We write this condition in the form

$$
\Delta r_{\text {flat }} \simeq 2\left|x_{\max }\right|
$$

where $\left|x_{\max }\right|$ is the largest radial excursion observed, at the considered time, among all the wave-trapped particles. We can estimate $x_{\max }$ by the following ansatz:

$$
x_{\max } \propto \frac{x_{\mathrm{sep}}}{\gamma T_{\mathrm{orb}}^{\mathrm{s}}}
$$

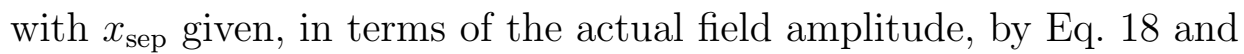

$$
T_{\text {orb }}^{\mathrm{s}} \equiv \lim _{\Theta_{0} \rightarrow 0} T_{\text {orb }}\left(\Theta_{0}\right)=\frac{2 \pi}{(|S| A)^{1 / 2}}
$$

We then obtain

$$
\Delta r_{\text {flat }} \simeq \frac{A}{\alpha \gamma}
$$

with $\alpha$ being a proportionality constant. The ansatz is justified a posteriori by numerically solving the system given by Eqs. 12, 13, 28, 29 and 34. Figure 20 compares the time evolution of $\Delta r_{\text {flat }}$, as defined by Eq. 31, with that yielded by Eq. 34 (with $\alpha=0.5$ ). The agreement between the two quantities is fairly good. 


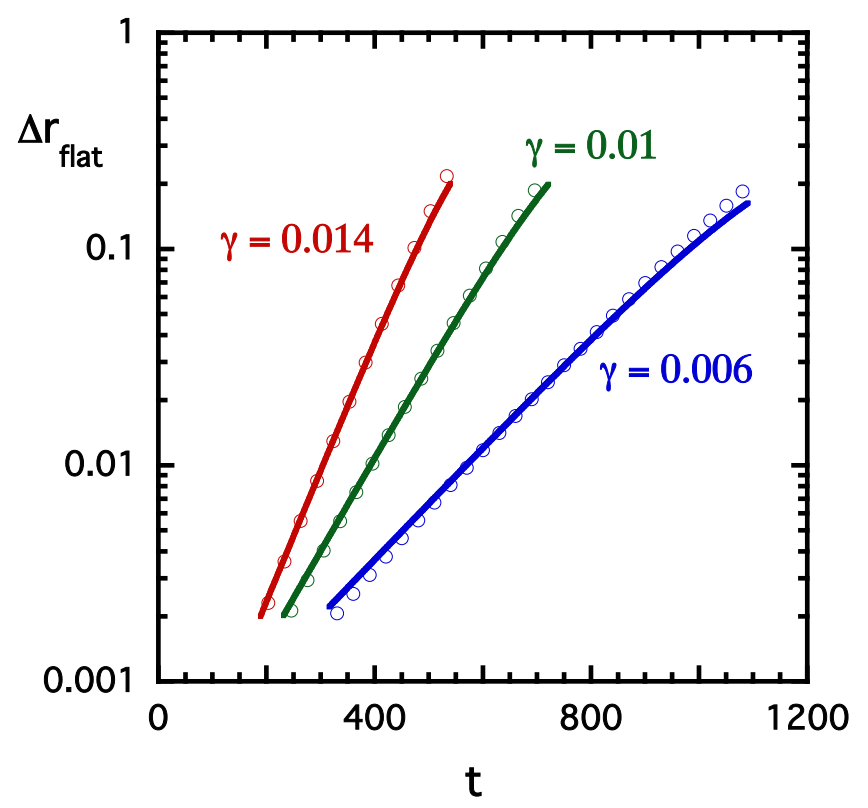

FIG. 20: Time evolution of $\Delta r_{\text {flat }}$, as defined by Eq. 31 (open circles) or Eq. 34 , with $\alpha=0.5$ (solid line). Three different values of $\gamma$ are considered.

Combining Eqs. 29 and 34 , we get

$$
\bar{\gamma}=\gamma\left\{1-\frac{A}{\alpha \gamma} \frac{1}{\min \left[\Delta r_{\text {mode }}, \Delta r_{\text {res }}\right]}\right\} \text {. }
$$

For $\Delta r_{\text {res }}<\Delta r_{\text {mode }}$, from Eq. 21 we get, for the saturation amplitude $(\bar{\gamma}=0)$

$$
A=\alpha \Delta r_{\text {res }} \gamma, \quad \text { with } \quad \Delta r_{\text {res }}=2 \gamma /|S| \text {, }
$$

analogous to Eq. 26. For $\Delta r_{\text {mode }}<\Delta r_{\text {res }}$, we find

$$
A=\alpha \Delta r_{\text {mode }} \gamma, \quad \text { with } \quad \Delta r_{\text {mode }}=\text { const }
$$

The predictions of this second qualitative model are consistent with the scalings shown in Fig. 19 and improve the previous ones (Eqs. 23 and 27) for radial decoupling. In particular, we see that the linear dependence on $\gamma$ is recovered for co-passing ions, as well as for counterpassing ones in the large $\gamma$ limit, where the mode saturation is due to radial decoupling $\left(\Delta r_{\text {mode }}<\Delta r_{\text {res }}^{-}\right)$. Moreover, the ratio between the saturation amplitude for co-passing and 
counter-passing ions is expected to be

$$
\frac{A^{+}}{A^{-}}=\frac{\Delta r_{\text {mode }}\left|S^{-}\right|}{2 \gamma}
$$

for moderate growth rates $\left(\Delta r_{\text {res }}^{-}<\Delta r_{\text {mode }}\right)$, and

$$
\frac{A^{+}}{A^{-}}=1
$$

in the large growth rate limit. We see that, for the reference case analysed in this paper, Eq. 38 would yield $A^{+} / A^{-} \simeq 3$, very close to the actual value given by simulations (cf. Fig. 13). The convergence of the two amplitude to the same value, Eq. 39, in the large $\gamma$ limit, on its side, reproduces very well simulation results (cf. Fig. 19).

\section{SUMMARY AND CONCLUSIONS}

In this paper, linear and nonlinear dynamics of $n=2$ BAEs driven unstable by anisotropic fast-ion populations (co-passing or counter-passing ions) has been investigated by single toroidal number numerical simulations performed by the hybrid MHD-particle code XHMGC. The linear-dynamics mode properties appear very similar for co-passing and counterpassing ion drive, with respect to radial structure, frequency and growth rate (slightly larger for counter-passing ions). On the other side, nonlinear dynamics exhibits significant differences: namely, saturation mode amplitude is larger, in the reference simulation considered, for the (slightly weaker) co-passing ion case. Furthermore, the scaling of such amplitude with the mode growth rate is linear, different from the quadratic scaling obtained, in the low growth rate limit, in the counter-passing ion case. These differences can be explained on the basis of the different radial profile of the resonance frequency in the two case: flat, for co-passing ions; steep, for counter-passing ions. Indeed, the radial region where the resonant interaction can take place is set by nonvanishing mode amplitude and matched resonance condition, $\left|\omega-\omega_{\text {res }}\right| \lesssim \gamma$. Each of these conditions is verified within a characteristic radial interval. Two regimes can be distinguished, depending on the relative size of the two intervals, $\Delta r_{\text {mode }}$ and $\Delta r_{\text {res }}$. In the first regime, the most stringent condition is given by the frequency matching $\left(\Delta r_{\text {res }}<\Delta r_{\text {mode }}\right)$; in the second regime, by the mode strength $\left(\Delta r_{\text {mode }}<\Delta r_{\text {res }}\right)$. The different radial dependence of $\omega_{\text {res }}$ for co-passing and counter-passing ions yields, for a given growth rate, $\Delta r_{\text {res }}^{-}<\Delta r_{\text {res }}^{+}$(at the same time, $\Delta r_{\text {mode }}$ is very similar 
for the two fast-ion distributions). In fact, in the reference case and, more generally, in the low growth rate limit, $\Delta r_{\text {res }}^{-}<\Delta r_{\text {mode }}<\Delta r_{\text {res }}^{+}$. In such limit, saturation, which is reached when the resonant-particle density flattening extends over the whole resonant-interaction width, corresponds to a flattening with of the order of the mode width, for co-passing ions; of the resonance width, for counter-passing ions. It has been shown that this difference in the saturation processes (called, respectively, radial decoupling and resonance detuning) accounts for the different saturation amplitude and its different scaling (linear vs quadratic) with increasing growth rate. For larger growth rates, even the resonance width for counterpassing ions becomes larger than the mode width, and a transition to the radial decoupling regime and the corresponding linear scaling is observed for the modes driven by such ions; saturation amplitudes tend to the same values for the two cases.

Note that the analysis presented in this paper does not encompass several aspects of Alfvén mode nonlinear dynamics, able to play an important role for different equilibria, like frequency chirping, large nonlinear modification of mode structure and synergic interaction of different toroidal number modes.

\section{APPENDIX}

In this Appendix we briefly recall some elements of the Hamiltonian mapping approach to the analysis of the nonlinear mode-particle dynamics. The selection of a suited test-particle sample has been described in Sect. III B. Following Ref. [19], we can represent such sample in the plane $\left(\Theta, P_{\phi}\right)$, with $\Theta \equiv \omega t+m \theta-n \phi$ being the wave-particle phase mapped into the interval $[0,2 \pi]$ (Fig. 21). At each time, each marker corresponds to the last equatorial-plane crossing $(\theta=2 \pi j$, with $j$ being an integer number) of a certain test particle; the marker changes positions, if needed, only after a new poloidal orbit has been completed. Each marker is doubled by a twin marker in the phase interval $[2 \pi, 4 \pi]$ in order to give a better visualisation of its dynamics. Marker colours (red or blue) depend on the initial $P_{\phi}$ value of each test particle and does not change with time. The boundary between two colours is initially fixed at $P_{\phi}=P_{\phi \text { res }}$ (corresponding to $r_{\text {res }}$, that is the position where $\omega_{\text {res }}=\omega$ and the wave phase $\Theta$ is a constant). During the whole linear phase, in which the field amplitude is so small that its effects on the particle orbits is negligible, $P_{\phi}$ is almost conserved. Then, during such phase (Fig. 21a), particle trajectories in the $\left(\Theta, P_{\phi}\right)$ plane essentially reduce 
to fixed points for $P_{\phi}=P_{\phi \text { res }}$, while they correspond to drift along the $\Theta$ axis in the positive/negative direction, for $P_{\phi}$ greater/less than $P_{\phi \text { res }}$. In the nonlinear phase, $P_{\phi}$ varies because of the mode-particle interaction (e.g., radial $\mathbf{E} \times \mathbf{B}$ drift). Even particles that were initially resonant are brought out of resonance, getting non zero $d \Theta / d t$ and drifting in phase until the drift in $P_{\phi}$ is inverted. Particles that cross the $P_{\phi}=P_{\phi \text { res }}$ line revert values of $d \Theta / d t$ as well. Thus, their orbits are bounded and they would properly close if the field amplitude were constant in time. This is true for particles born close to the resonance, while particles born with $P_{\phi}$ far from the resonance maintain drifting orbits, as they do not cross $P_{\phi}=P_{\phi \text { res }}$. Bounded orbits and drifting ones are divided by an instantaneous separatrix, whose $P_{\phi}$ width increases with increasing field amplitude (Fig. 21b).

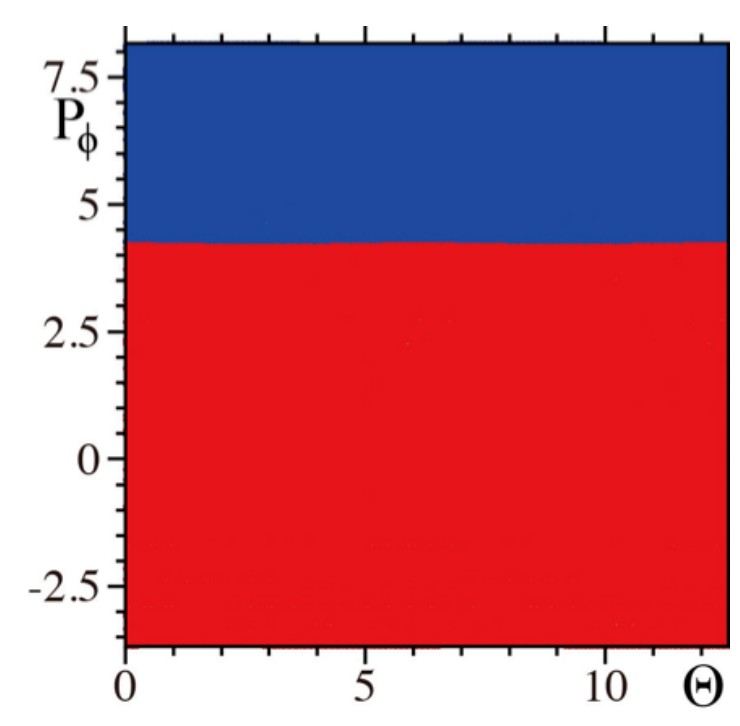

(a) linear phase

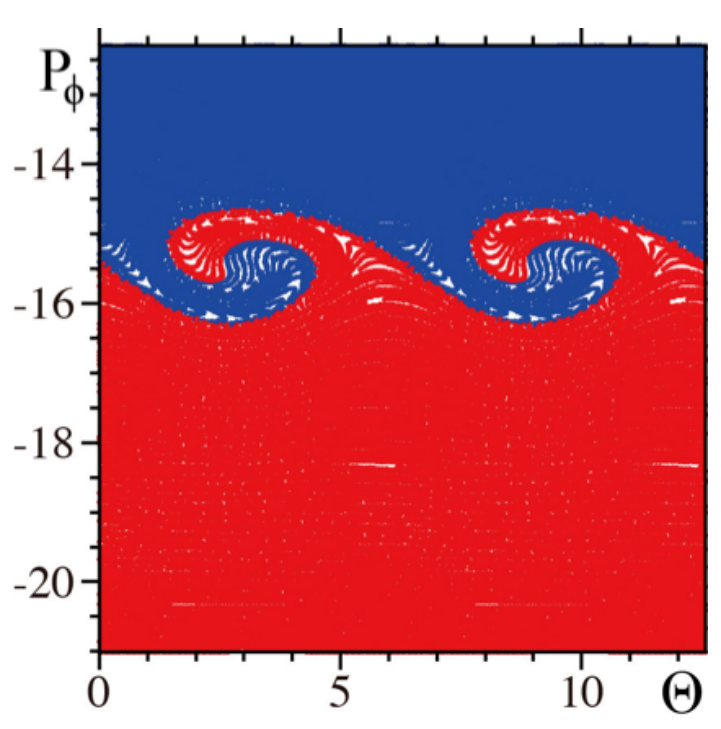

(b) nonlinear phase

FIG. 21: Representation of the whole test-particle sample in the plane $\left(\Theta, P_{\phi}\right)$, at certain times in the linear (a) and nonlinear (b) phase. The position of each marker corresponds to the last equatorial-plane crossing of a test particle. Colours (red or blue) depends on the initial $P_{\phi}$ value of each test particle and does not change with time. The boundary between two colours is initially fixed at $P_{\phi}=P_{\phi \text { res }}$, corresponding to the position where $\omega_{\text {res }}=\omega$. The formation, in the nonlinear phase, of bounded orbits separated from drifting orbits by an instantaneous separatrix can be seen. 


\section{ACKNOWLEDGEMENTS}

The authors would like to thank G. Vlad and G. Fogaccia, co-developers of the XHMGC code, for precious support in the simulation work and helpful comments. This work has been carried out within the framework of the EUROfusion Consortium and has received funding from the Euratom research and training programme 2014-2018 under grant agreement No. 633053. The views and opinions expressed herein do not necessarily reflect those of the European Commission. One of the authors, X. Wang, would like to acknowledge the Project Supported by Open Research Program from Key Laboratory of Basic Plasma Physics, Chinese Academy of Sciencs (CAS) for some initial work in this paper.

[1] L. Chen and F. Zonca, Nucl. Fusion 47, S727 (2007).

[2] L. Chen and F. Zonca, Phys. Plasmas 20, 055402 (2013).

[3] Ph. Lauber, Phys. Rep. 533, 33 (2013).

[4] N. N. Gorelenkov and S. D. Pinches and K. Toi, Nucl. Fusion 54, 125001 (2014).

[5] S. D. Pinches and I. T. Chapman and Ph. W Lauber and H. J. C Oliver, S. E. Sharapov, K. Shinohara and K. Tani, Phys. Plasmas 22, 021807 (2015).

[6] L. Chen and F. Zonca, "Physics of Alfvén waves and energetic particles in burning plasma", Rev. Mod. Phys., submitted (2014).

[7] B. N. Breizman and S. E. Sharapov, Plasma Phys. Control. Fusion 53, 054001 (2011).

[8] S. E. Sharapov et al., Nucl. Fusion 53, 104022 (2013).

[9] F. Zonca et al., Plasmas Phys. Control. Fusion 57, 014024 (2015).

[10] C.Z. Cheng, L.Chen and M.S. Chance, Ann. Phys. 16121 (1985).

[11] L. Chen,Phys. Plasmas 11519 (1994).

[12] H.L. Berk and B.N. Breizman, Phys. Fluids B 22246 (1990).

[13] H. L. Berk, B. Breizman and M. Pekker, Phys. Rev. Lett. 76 1256, (1996)

[14] H.L. Berk, B.N. Breizman and N.V. Petiashivili, Phys. Lett. A 234213 (1997).

[15] H.L. Berk, B.N. Breizman, J. Candy, M. Pekker, and N. V. Petiashvili, Phys. Plasmas 6, 3102 (1999).

[16] H.L. Berk, B.N. Breizman and M. S. Pekker, Plasma. Phys. Rep. 23 778, (1997). 
[17] B. N. Breizman, H. L. Berk, M. Pekker, F. Porcelli, G. V. Stupakov, and K. L. Wong, Phys. Plasmas 4, 1559 (1997).

[18] F. Zonca et al., Nucl. Fusion 45477 (2005).

[19] S. Briguglio et al., Phy. Plasmas 21112301 (2014).

[20] F. Zonca et al., New J. Phys. 17013052 (2015).

[21] W. W. Heidbrink, E. J. Strait, M. S. Chu and A. D. Turnbull, Phys. Rev. Lett 71, 855 (1993).

[22] Ph. Lauber et al, Plasma Phys. Control. Fusion 51124009 (2009).

[23] R. Nazikian et al, Phys. Rev. Lett. 96105006 (2006).

[24] F. Zonca, L. Chen, J. Q. Dong and R. A. Santoro, Phys. Plasmas 61917 (1999).

[25] L. Chen, R. B. White and M. N. Rosenbluth, Phys. Rev. Lett. 521122 (1984).

[26] H. Biglari and L. Chen, Phys. Rev. Lett. 673681 (1991).

[27] S. T. Tsai and L. Chen, Phys. Fluids B 53284 (1993).

[28] L. Chen and F. Zonca, Physica Scripta T60 81 (1995).

[29] C. Cheng, N. Gorelenkov and C. Hsu, Nucl. Fusion 351639 (1995).

[30] F. Zonca, L. Chen and R. A. Santoro, Plasma Phys. Control. Fusion 382011 (1996).

[31] W. Heidbrink, E. Carolipio, R. James and E. Strait,Nucl. Fusion 351481 (1995).

[32] W. Heidbrink et al, Phys. Plasmas 61147 (1999).

[33] F. Nabais et al Phys. Plasmas 12102509 (2005).

[34] E. D. Fredrickson et al Phys. Plasmas 13056109 (2006).

[35] F. Zonca et al Nucl. Fusion 471588 (2007).

[36] D. Darrow et al Nucl. Fusion 48084004 (2008).

[37] M. Podesta et al, Phys. Plasmas 16056104 (2009).

[38] E. D. Fredrickson et al Phys. Plasmas 16122505 (2009).

[39] F. Zonca et al Nucl. Fusion 49085009 (2009).

[40] X. Wang et al., Plasma Phys. Control. Fusion 52115005 (2010).

[41] F. Zonca and L. Chen, Plasma Phys. Control. Fusion 48, 537 (2006).

[42] F. Zonca and L. Chen, Phys. Plasmas, 21, 072120, (2014).

[43] F. Zonca and L. Chen, Phys. Plasmas, 21, 072121, (2014).

[44] S. Briguglio, G. Vlad, F. Zonca and C. Kar, Phys. Plasmas 23711 (1995).

[45] S. Briguglio, F. Zonca and G. Vlad, Phys. Plasmas 53827 (1998).

[46] X. Wang et al., Phys. Plasmas 18052504 (2011). 
[47] Ph. Lauber et al., J. Comp. Phys., 226(1) 447 (2007)

[48] M. Abramowitz and I.A. Stegun. Handbook of Mathematical Functions. Dover, 1972. 